\title{
Erratum: A bacterial platform for fermentative production of plant alkaloids
}

Akira Nakagawa, Hiromichi Minami, Ju-Sung Kim, Takashi Koyanagi, Takane Katayama, Fumihiko Sato \& Hidehiko Kumagai

Nature Communications 2:326 doi: 10.1038/ncomms1327 (2011); Published 24 May 2011; Updated 22 May 2012.

In Fig. 2 of this article, the directions of the arrows associated with 6OMT and CNMT were inadvertently changed during the production process. The correct version of the figure appears below:

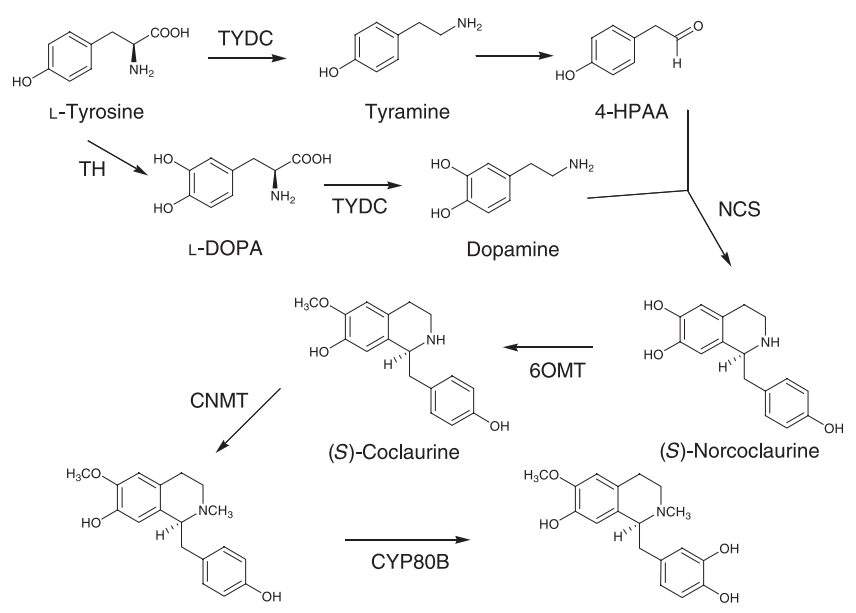

(S)-N-Methylcoclaurine

(S)-3'-Hydroxy- $N$-methylcoclaurine



